\title{
PAULO FREIRE, LAS PEDAGOGÍAS POST-CRÍTICAS Y EL DILEMA PEDAGÓGICO
}

\author{
Paulo Freire, post-critical pedagogies, \\ and the pedagogical dilemma
}

\author{
Andrés MEJÍA DELGADILLO \\ Universidad de los Andes. Colombia. \\ jmejia@uniandes.edu.co \\ bttps://orcid.org/0000-0002-3968-9410
}

Fecha de recepción: 17/02/2020

Fecha de aceptación: 25/03/2020

Fecha de publicación en línea: 01/07/2020

\section{RESUMEN}

El Manifiesto para una Pedagogía Post-crítica de Hodgson, Vlieghe y Zamojski comparte varios elementos con las propuestas que, con el mismo nombre, circulan desde finales de la década de 1980. Dos de los elementos con los que estos autores pretenden distanciarse de la pedagogía crítica, y que me interesan aquí en particular, son la propuesta de asumir la igualdad entre educadores y educandos como un punto de partida —en lugar de la superioridad de los primeros sobre los segundos- y la de abstenerse de proponer compromisos sustantivos sobre un futuro utópico hacia el cual se debe avanzar y que sirve para juzgar el presente. Al analizar la obra del autor posiblemente más importante de la pedagogía crítica, Paulo Freire, señalo que un afán de igualdad ya se encontraba en su obra de manera explícita y contundente. A la vez, sin embargo, también existen en ella varios elementos relacionados con un afán de justicia y dignidad más allá de la relación pedagógica —las ideas de conciencia crítica y concienciación - que, de modo contradictorio, materializan un principio de desigualdad. Los proponentes del Manifiesto para una Pedagogía Post-crítica definen de antemano un principio de igualdad entre educadores y educandos, con lo cual terminan evadiendo una de las dimensiones de la responsabilidad de cuidado que corresponde a la práctica educativa de la pedagogía crítica. Propongo que, al tomar 
la educación crítica como una práctica de cuidado, ninguno de estos afanes podrá evadirse. Concluyo que una pedagogía crítica se constituye en la atención siempre presente y profunda hacia las necesidades de los educandos, en la tensión entre un afán de igualdad y un afán de justicia y dignidad.

Palabras clave: Paulo Freire; pedagogía crítica; pedagogía post-crítica; igualdad; dilema pedagógico.

\section{ABSTRACT}

The Manifesto for a Post-Critical Pedagogy by Hodgson, Vlieghe and Zamojski shares several elements with the proposals that, with the same name, have been circulating since the late 1980s. Two of the elements with which these authors seek to distance themselves from critical pedagogy, and which are of particular interest to me here, are the proposal to assume equality between educators and educands as a starting point — rather than the superiority of the former over the latter - and to refrain from proposing substantive commitments about a utopian future towards which one must advance and which serves to judge the present. When analysing the work of the possibly most important author of critical pedagogy, Paulo Freire, I point out that a will to equality was already explicitly and strongly in his work. At the same time, however, there are also several elements related to a will to justice and dignity beyond the pedagogical relationship - the ideas of critical conscience and awareness - that, in a contradictory way, materialise a principle of inequality. The proponents of the Manifesto for a Post-Critical Pedagogy define in advance a principle of equality between educators and educands, thereby ending up evading one of the dimensions of responsibility for care that corresponds to the educational practice of critical pedagogy. I propose that, by taking critical education as a caring practice, none of these endeavours should be renounced. I conclude that a critical pedagogy is constituted in the always present and deep attention to the needs of the students, in the tension between a will to equality and a will to justice and dignity.

Key words: Paulo Freire; critical pedagogy; post-critical pedagogy; equality; pedagogical dilemma.

\section{LAS PEDAgogías POSTCRíticas ANTES Y AHORA}

Naomi Hodgson, Joris Vlieghe y Piotr Zamojski han producido y publicado su Manifiesto para una Pedagogía Post-crítica ${ }^{1}$, formulándolo como un conjunto de

principios fundados en la creencia de la posibilidad de transformación, tal y como se establece en la teoría y la pedagogía críticas, pero con una actitud afirmativa: una

1. La versión española que se cita en este artículo forma parte del monográfico «Una respuesta iberoamericana al 'Manifiesto por una pedagogía post-crítica'»: http://dx.doi.org/10.14201/teri.22862 
orientación post-crítica hacia la educación que se beneficia de las condiciones actuales y que se funda en la esperanza de lo que aún está por venir.

Algo de lo que los autores son conscientes es el riesgo que toman al proponer este Manifiesto en unos tiempos de sospecha generalizada frente a la formulación de principios normativos que puedan operar como una utopía que se imponga a otros - con las violencias que esto trae, tanto de orden epistémico (Spivak, 1988; Segato, 2016) como de otros tipos- Yo creo que, por esta misma razón, es muy valiente hacerlo, y que su primer principio es por ello tal vez el más valioso de todos: «El primer principio que aquí se establece es, simplemente, que hay principios que defender». La idea de que la educación es comprometida y no indiferente frente a lo que ocurre y lo que puede ocurrir.

Al mismo tiempo, los autores del Manifiesto no especifican con qué se compromete la educación, sustantivamente, más allá de unos principios generales que de ninguna manera van a dibujar una imagen de un futuro utópicamente feliz. Por eso, para ellos, esto,

(...) en sí mismo, no nos compromete con nada más allá, es decir, no nos compromete a tener que hacer "X». No se trata de afirmar la normatividad en el sentido de definir un estado ideal, presente o futuro, contra el cual debiera juzgarse la práctica actual.

Este gesto de evitar un compromiso sustantivo se relaciona con otro que, según mi interpretación, es constitutivo de uno de los puntos más centrales de la propuesta contenida en el Manifiesto: no abandonar, mantener, una educación comprometida como la de las pedagogías críticas tradicionales, pero esta vez adoptando una visión de corte rancièriano de nuestras relaciones con los otros: una que no asuma la superioridad del educador crítico sobre el educando, y que por lo tanto no entienda que el educador crítico es quien, desde un lugar de mayor altura, puede ver el mundo como realmente es para así mostrárselo al educando ingenuo para que este pueda salir de su ingenuidad. En su lugar, esta propuesta parte de la igualdad: una actitud que adopte la igualdad de inteligencias entre educador y educandos (Rancière, 2003) como un principio que ponemos en práctica más que indagar si efectivamente era cierto. En palabras de los autores del Manifiesto, «el tercer principio se basa, pues, en asumir que la igualdad (...) y la posibilidad de transformación - a nivel individual y colectivo - conllevan un giro desde una

\section{pedagogía crítica a una pedagogía post-crítica».}

Comienzo mi contribución señalando que hay varios sentidos en los que una discusión de naturaleza similar se ha planteado ya con anterioridad, y que incluso la expresión misma "pedagogía postcrítica» también cuenta con una historia de varias 
décadas ${ }^{2}$. Ya en 1988, Elizabeth Ellsworth presentaba en un congreso una crítica muy significativa a la pedagogía crítica en un texto que sería publicado al año siguiente con el título Why Doesn't This Feel Empowering? Working Through the Repressive Myths of Critical Pedagogy. Allí, al referirse a un curso universitario dictado por ella sobre pedagogías antiracistas y medios de comunicación, esta autora señala que sus «esfuerzos de poner en práctica los discursos de la pedagogía crítica nos llevaron a reproducir relaciones de dominación en nuestro salón de clases», y que "dichos discursos estaban 'trabajando a través' de nosotros de modos represivos y se habían convertido en sí mismos en vehículos de represión" (Ellsworth, 1989, p. 298). La pregunta que ella lanza en ese momento a los educadores críticos es: "¿Qué diversidad silenciamos en nombre de una pedagogía 'liberadora'?» (Ellsworth, 1989, p. 299). Este artículo tuvo una amplia discusión —incluidas reacciones no muy amigables de parte de teóricos líderes de la pedagogía crítica (p. ej., Giroux, 1989) - y se puede argüir que fue influyente en algunas iniciativas que algunos luego llamaron "pedagogías postcríticas» o "pedagogías postradicales» (Rattansi y Reeder, 1992; Green, 1998; Mejía, 2009).

Lo interesante de esta discusión es su problematización de la posición superior a la vez que neutral u objetiva que puede asumir el educador crítico, y que lo autoriza para guiar a sus estudiantes hacia una conciencia crítica que éstos no han desarrollado aún, pero él sí. Este modo de plantear la situación educativa, así como la relación entre educador y educandos deja a estos últimos en una situación dilemática ${ }^{3}$ que, como lo plantea Buckingham, ocurre cuando los estudiantes rechazan la emancipación que se les ofrece (1998, p. 5):

¿Qué pasa si no quieren ser 'liberados' o 'empoderados' como el maestro lo ha imaginado? Un modo familiar de resolver este dilema es apelar a una noción de falsa conciencia; dado que se les ve como de alguna manera confundidos o engañados, los estudiantes son incapaces de darse cuenta de sus verdaderos intereses y el maestro debe por tanto actuar por ellos. Este enfoque tiene la ventaja de ser un argumento autoconfirmatorio: entre más se resistan los estudiantes, más necesitan de una cura ideológica y más se justifican la intervención y la autoridad del maestro.

Quiero señalar aun otro elemento presente en esa crítica que las propuestas de pedagogías postcríticas ya hacían hace más de tres décadas, que parece resonar

2. Aquí me refiero tanto a la expresión "pedagogía postcrítica» como a su similar "pedagogía postradical", las cuales se definen en referencia a las normalmente intercambiables "pedagogía crítica" y "pedagogía radical».

3. Uso aquí la palabra "dilemática» en el sentido rescatado por Lyotard y que él ilustra con la brillante anécdota del caso del maestro sofista Protágoras y su discípulo y aprendiz en las artes de la retórica, Euathlus: «Se cuenta la historia de cuando Protágoras exigió a Euathlus sus honorarios. Euathlus se negó a pagar, diciendo: ‘Pero no he ganado aún mi primera victoria!'. Protágoras replicó: 'Pero si yo gano esta disputa, me deberás pagar porque habré ganado, y si tú ganas, me deberás pagar porque tú habrás ganado'» (Lyotard, 1988, p. 6). 
con el ánimo del primer principio del Manifiesto de Hodgson, Vlieghe y Zamojski cuando sugieren que no pretenden «definir un estado ideal, actual o futuro, contra el cual debiera juzgarse la práctica actual». Así, ya Ellsworth proponía evitar principios que de forma sustantiva nos remitieran a un futuro utópico general, universal. Por el contrario, al describir su práctica pedagógica (postcrítica), ella reportaba (1989, pp. 298-299):

'Trabajamos a través' y 'hacia afuera' del lenguaje abstracto de la literatura [de la pedagogía crítica] ('mitos') sobre quiénes 'deberíamos' ser y qué 'debería' estar ocurriendo en nuestra clase, y nos movimos hacia prácticas de aula que eran específicas para el contexto y que parecían responder de manera mucho más atenta a nuestras propias comprensiones de nuestras identidades y situaciones sociales.

Mi intención con este regreso al pasado no es demeritar o descalificar el Manifiesto de Hodgson, Vlieghe y Zamojski, acusándolo de falta de originalidad o de obsolescencia. No creo que sea necesario pedirle a un manifiesto que sea completamente novedoso; un manifiesto simplemente declara un conjunto de principios a los que sus formuladores declaran que quieren ceñirse. Lo original en este caso, incluso, está en su presentación en forma de manifiesto. Por otro lado, de ninguna manera estimo obsoleto lo que allí se plantea. Creo que es pertinente en la actualidad, lo cual puede verse en su alineación grosso modo con un conjunto amplio de tendencias contemporáneas que se han preocupado por varios de los elementos expresados por Hodgson, Vlieghe y Zamojski (en la línea de autores como Boaventura Santos, Jacques Rancière, y Slavoj Žižek, o, en la filosofía de la educación en particular, Gert Biesta y Jan Masschelein). En lugar de eso, simplemente quiero señalar que en el tiempo que ha pasado desde las pedagogías postcríticas de antes hasta las de ahora se han dado algunas discusiones que vale la pena retomar alrededor de lo que — casi en forma de trabalenguas- podríamos llamar las críticas postcríticas a la pedagogía crítica.

En concreto, me referiré en este artículo a dos de los elementos relacionados entre sí que están en juego aquí y que ya resumí brevemente antes: la igualdad o desigualdad entre educador y educandos, y la definición previa, o no, de un futuro utópico deseado desde el cual se juzga el presente, y que los educadores conocen mejor que los educandos. Estos dos elementos están conectados entre sí a través del hecho de que la teorización de un futuro utópico y de la diferencia con el presente es lo que brinda a los educadores críticos un nivel de superioridad sobre sus educandos, desde el cual se legitima su intervención pedagógica.

Examinaré, entonces, la aparición de estos elementos en la pedagogía crítica. En concreto, abordaré las maneras en las que los dos elementos opuestos de estos pares binarios —igualdad y desigualdad - aparecen allí. Ahora bien, dado que el corpus de literatura de este enfoque educativo es enorme, me concentraré en la obra de uno de sus autores más prolíficos e importantes — si no el más importante-, que además es considerado fundacional para dicho movimiento: Paulo Freire. De 
este modo, me aseguro de contar con una base amplia y profunda de ideas a la vez que puedo examinar de manera más directa un conjunto de elementos considerados canónicos dentro del movimiento.

\section{La igualdad en la Relación educativa, en la pedagogía crítica de Paulo FREIRE}

Comienzo por decir que Paulo Freire ya era muy consciente de algunos de los asuntos que preocupan a los autores de las pedagogías postcríticas tanto de antes como de ahora. La pedagogía crítica freireana declara muy explícitamente, y en numerosas ocasiones, un compromiso con lo que podríamos llamar un afán de igualdad entre educadores y educandos. Esto se configura en al menos dos niveles. En el primero aparece en un desmarcamiento de la idea de algunos teóricos marxistas para quienes hay una división de trabajo en la revolución que busca una sociedad más justa: por un lado, unos intelectuales que sí pueden ver la realidad críticamente, tal como es, serían las cabezas pensantes; y, por el otro lado, el pueblo, que no comprende suficientemente lo que está en juego como para poder otorgarle una voz legítima y una influencia en el curso de acción, y que por ello debe limitarse a seguir a sus líderes intelectuales (Freire, 1969/1997 y 1970/2000). Para él, la lucha por un futuro mejor no puede darse por medio de unos intelectuales que piensan por el pueblo. En vez de esto, los individuos deben dejar de ser objetos de la historia, llevados por las corrientes de sus épocas, para convertirse en sujetos históricos que desarrollan agencia para transformar la realidad a su alrededor.

Este elemento es fundamental para justificar la necesidad de la educación política que se define en la pedagogía crítica: es allí donde va a ser posible configurar los espacios en donde el pueblo va a pensar críticamente su realidad. La pedagogía crítica no sería necesaria si la consigna fuera la de que el pueblo no tiene la necesidad de pensar por sí mismo, ya que los intelectuales van a pensar por él. Es por esto por lo que planteo aquí que el interés en una pedagogía crítica, en sí mismo, ya es expresión de afán de igualdad ${ }^{4}$.

Pero hay un segundo nivel en el que, propongo, también aparece en la propuesta freireana un afán de igualdad. Uno de los elementos más reconocidos de

4. Aunque no es directamente pertinente para lo central de mi discusión, quiero señalar una acusación problemática que los autores del manifiesto hacen a la pedagogía crítica y que valdría la pena explicar mejor: "termina siendo un ethos desesperanzado - por no decir cínico-, como resultado de lo aparentemente ineludible que resulta ser la racionalidad neoliberal.» Pero se puede decir que, si en algo se basa la pedagogía crítica, es en la esperanza. En esto rompe con varios autores de la tradición de la sociología crítica de la educación y sus teorías de la reproducción social (ver Giroux, 1983). La esperanza aparece en el centro de la pedagogía crítica tanto desde los anhelos de revolución que marcan los trabajos más tempranos de Freire como en sus obras más tardías, una de los cuales incluso se titula Pedagogía de la esperanza (1993). 
su teorización proviene de la discusión que hace Freire sobre la educación bancaria (1970/2000). Para él, así como la educación puede ser crítica, también puede actuar para perpetuar la dominación y la opresión. Para este segundo fin, se vale dos mecanismos. Por un lado, temáticamente, al no problematizar la realidad —no cuestionar el orden social con su injusticia y su opresión- se transmiten conocimientos a los estudiantes que simplemente sirven para alimentar el orden social existente: por ejemplo, conocimientos técnicos útiles para adaptarse al mercado laboral. Y, por el otro lado, se transmite a los estudiantes una noción del rol social que deben ocupar, en el cual ellos aprenden a no cuestionar, a considerarse a sí mismos ignorantes, a no pensar sino dejar que otros piensen por ellos. Podemos pensar aquí en una especie de currículo oculto. Dada la coincidencia del afán de igualdad freireano con la preocupación expresada en el Manifiesto para una pedagogía post-crítica, vale la pena traer aquí la lista de principios que subyacen y orientan la educación bancaria (2000, p. 74):

a) El educador es siempre quien educa; el educando el que es educado.

b) El educador es quien sabe; los educandos quienes no saben.

c) El educador es quien piensa, el sujeto del proceso; los educandos son los objetos pensados.

d) El educador es quien habla; los educandos quienes escuchan dócilmente.

e) El educador es quien disciplina; los educandos los disciplinados.

f) El educador es quien opta y prescribe su opción; los educandos quienes siguen la prescripción.

g) El educador es quien actúa; los educandos son aquellos que tienen la ilusión de que actúan, en la actuación del educador.

h) El educador es quien escoge el contenido programático; los educandos, a quienes jamás se escucha, se acomodan a él.

i) El educador identifica su autoridad del saber con la autoridad funcional, la que opone antagónicamente a la libertad de los educandos. Son éstos quienes deben adaptarse a las determinaciones de aquel.

j) Finalmente, el educador es el sujeto del proceso; los educandos, meros objetos.

Precisamente, Freire opone a esta idea bancaria una educación dialógica problematizadora, la cual es una educación crítica. En ella interactúan los educadoreseducandos con los educandos-educadores en un tipo de diálogo que se caracteriza, entre otras cosas, por el amor y la humildad. Más aun, para Freire, sin humildad no puede haber diálogo (2000, p. 73):

La pronunciación del mundo, con la cual los hombres lo recrean permanentemente, no puede ser un acto arrogante. (....) ¿Cómo puedo dialogar, si alieno la ignorancia, esto es, si la veo siempre en otro y nunca en mí? ¿Cómo puedo dialogar, si me admito como un hombre diferente, virtuoso por herencia, frente a los otros, meros objetos en 
quienes no reconozco otros 'yo'? ¿Cómo puedo dialogar, si me siento participante de un 'guetto' de hombres puros, dueños de la verdad y del saber, para quienes todos los que están fuera son 'esa gente' o son 'nativos inferiores'?

Para concluir esta sección, traigo aquí dos frases que encuentro claves para notar la fuerza con la que Freire expresa este afán de igualdad. La primera de ellas aparece justo en el cierre de su obra magna, Pedagogía del Oprimido (2000, p. 240, énfasis añadido): "Si nada queda de estas páginas, esperamos que por lo menos algo permanezca: nuestra confianza en el pueblo. Nuestra fe en los hombres y en la creación de un mundo en el que sea menos difícil amar.» La segunda se constituye en una especie de leitmotiv que varía levemente en cada una de sus apariciones. La que aquí presento es la que se encuentra como tema descriptor del primer capítulo de esa misma obra (p. 23, énfasis añadido): "Nadie libera a nadie, ni nadie se libera solo. Los hombres se liberan en comunión».

Es decir, Freire es plenamente consciente de los problemas que trae una educación bancaria; es consciente de que una educación bancaria sería completamente contraria al ideal pedagógico-político de la pedagogía crítica. Es interesante que los autores del Manifiesto declaran que "Aquí partimos de la asunción de que podemos hablar y actuar - juntos - y, por lo tanto, pasar de la pedagogía hermenéutica que conlleva la pedagogía crítica, a defender una - segundo principio- hermenéutica pedagógica». Creo que sería difícil, a partir de lo que he mostrado hasta aquí, aseverar que no existe en la propuesta freireana un fuerte propósito de hablar y actuar juntos.

\section{La desigualdad en la Relación educativa, EN la PEdagogía Crítica de Paulo Freire}

La discusión en la sección anterior parecería llevarnos a concluir que los proponentes de una pedagogía postcrítica, tanto los de antes como los de ahora, son culpables de un enorme error de juicio al pasar por alto el hecho de que la pedagogía crítica se ocupa de la igualdad. Pero, por supuesto, las cosas no son tan simples. La pedagogía crítica, incluida la freireana, puede ser también culpable de intentar imponer una visión de un futuro utópico único, universal y por lo tanto descontextualizado, no situado (como sugería Ellsworth, 1989), como punto final al que todos los estudiantes deberían llegar. Y el conocimiento privilegiado de ese futuro utópico, así como de la brecha existente entre el presente y ese futuro, daría a los educadores una posición de superioridad que serviría para legitimar que ellos adopten el papel de develar para los estudiantes aquello que éstos son incapaces de ver por su cuenta. Esto, como bien lo señalan los proponentes de una pedagogía postcrítica, puede constituir una forma de violencia.

Podemos ver la operación de esto en uno de los principales recursos teóricos usados por la pedagogía crítica, el cual formula lo que puede entenderse como el fin de la educación freireana: la conciencia crítica. A partir de ésta se definen, 
además, los estadios del proceso por el que pasan los estudiantes para llegar allí, el cual se denomina concienciación (conscientizaçao) (Freire, 1997): en el estadio más básico se tiene una conciencia semiintransitiva; de allí se pasa a una conciencia transitiva ingenua, y de ahí finalmente se llega a una conciencia transitiva crítica. En este proceso, la preparación comienza por ayudar al estudiante a sacar al opresor de adentro, a darse cuenta de la injusticia y la opresión, y en algún sentido desdomesticar su conciencia. Para mostrar aún más claramente la condición de desigualdad presente en esta propuesta, vale la pena nombrar las varias expresiones que Freire usa para referirse al estado en el que se encuentran los educandos antes de la educación crítica, y que denotan su condición inferior: "conciencia ingenua" (1997), "falsa percepción", "falsa conciencia", "conciencia sumergida" y "mente mitológica» (2000), entre otras.

Esto quiere decir que el primer nivel en el que aparece el afán de igualdad en la propuesta freireana al que me referí en la sección anterior, y que da sentido a la idea misma de que haya una pedagogía crítica, no es suficiente: aún es posible que los espacios educativos puedan ser configurados de modo que lo que se ocurra allí sea que los educadores críticos (o los intelectuales) busquen llevar a los educandos (o el pueblo) del nivel inferior en el que se encuentran a convertirse en los primeros, a través de desarrollar una conciencia crítica.

¿Por qué, entonces, insistiría la pedagogía crítica freireana en el desarrollo de una conciencia crítica como el fin de su propuesta educativa? El motor mayor de la pedagogía crítica son la justicia y la dignidad, y promover procesos de concienciación tiene que ver directamente con el reconocimiento de las formas existentes de injusticia e indignidad, tanto directas como estructurales. Se puede decir que la pregunta pedagógica aquí es cómo hacer para que los educandos comprendan de manera profunda y crítica las formas de opresión a las que están siendo sometidos, así como las formas estructurales que producen, mantienen o refuerzan dichas formas de opresión. La idea de desarrollar una conciencia crítica proviene, por tanto, de un afán de justicia y dignidad más amplio, que va más allá de la relación entre educador y educandos para ocuparse de la sociedad más allá. Como vimos antes, Ellsworth (1989) y otros autores han sugerido que este es un ideal abstracto y descontextualizado en la pedagogía crítica. Pero hay que tener cuidado con esta afirmación: esta idea de lo abstracto parecería implicar que el educador crítico mantiene un interés solamente intelectual por sus educandos, como instancias particulares del contrario de su ideal abstracto de justicia y equidad. Es decir, esa afirmación sugeriría que el educador crítico no profesa un genuino amor por sus educandos, una preocupación por cada uno de ellos como seres que merecen desarrollar una vida mejor. Si bien no pretendo refutar esta posible implicación a través de la argumentación, creo que es justo decir que tampoco es posible aseverarla. Dejo al menos, entonces, abierta la posibilidad de que el amor directo y genuino hacia sus educandos sea parte del 
ethos de muchos educadores críticos en su afán de justicia y dignidad. Aunque a veces puedan equivocarse en su intento.

\section{4. ¿Y ENTONCES?}

He intentado mostrar que al menos algunas de las preocupaciones más centrales de los proponentes de una pedagogía postcrítica — aquellas por la igualdad y por la necesidad de ubicarse en lo concreto, de manera situada - también preocupaban y ocupaban el trabajo de la pedagogía crítica de Paulo Freire. Pero al mismo tiempo intenté mostrar que el modo en que orientan su afán de justicia y dignidad también puede traicionar esos postulados de igualdad. Varios autores han señalado esta contradicción o tensión en la propuesta freireana, en particular, y en la pedagogía crítica, en general (ver Taylor, 1993; Burbules; 1993, 1995; Mejía, 2004).

Es importante notar que, al menos en mi lectura de la obra de Paulo Freire y de otros autores de la pedagogía crítica, yo no encuentro una tematización suficiente y profunda de esta tensión, ni una reflexión sobre cómo lidiar con ella en la práctica. Como si fuera posible y poco problemático reconciliar las dos cosas: como si, dejados a su propio razonamiento crítico y enfocándose en su propia situación concreta y particular, los educandos llegaran necesariamente a las mismas comprensiones de su mundo que los educadores críticos (Mejía, 2004). Y entonces la tensión permanece oculta, en una especie de actitud de evasión. Por ello, creo que es muy valioso el llamado que nos hacen las pedagogías postcríticas, tanto las de antes como las de ahora.

¿Hemos entonces de adoptar la propuesta de corte rancièriano expresada en el Manifiesto de Hodgson, Vlieghe y Zamojski, al otro lado del espectro? ¿Resuelve la pedagogía postcrítica el problema? Creo que si aceptamos que no es gratuito que en la pedagogía crítica freireana aparezca esa tensión entre el afán de igualdad y el afán de justicia y dignidad, entonces tampoco podríamos concluir eso. Como lo argumento más adelante, la postura postcrítica constituye también una especie de evasión, solo que una distinta.

Para abordar esto, recurro ahora a una idea planteada al final de la sección pasada: la de la relación educativa entre educador y educandos como una relación basada en el amor. Es de notar que el amor es constitutivo de la propuesta freireana (2000, pp. 102-103):

Es así como no hay diálogo si no hay un profundo amor al mundo y a los hombres. (....) Dondequiera exista un hombre oprimido, el acto de amor radica en comprometerse con su causa. La causa de su liberación. Este compromiso, por su carácter amoroso, es dialógico.

El amor implica una valoración de aquello amado en sí mismo, un reconocimiento de su valor intrínseco y de ahí la asunción emotiva del compromiso por su cuidado (Callan, 2006). El amor — distinto del enamoramiento posesivo en muchas relaciones de pareja- conlleva una orientación de cuidado hacia el otro, hacia lo amado. Mi propuesta es entender la práctica educativa de la pedagogía crítica como 
una práctica de cuidado, en el sentido más general propuesto por Noddings (2003). De hecho, el cuidado parece ser central para los autores del Manifiesto: «vemos la tarea de la pedagogía postcrítica no como un esfuerzo por desenmascarar sino por proteger y por cuidar».

Tronto propone entender el cuidado como "una especie de actividad que incluye todo lo que hacemos para mantener, contener y reparar nuestro 'mundo' de modo que podamos vivir en él tan bien como sea posible. Ese mundo incluye nuestros cuerpos, nosotros mismos, y nuestro entorno» (2006). Tronto adicionalmente propone cuatro elementos centrales del cuidado: (1) atención (attentiveness), o la disposición a darse cuenta de la necesidad; (2) responsabilidad, o la voluntad de responder a y cuidar esa necesidad; (3) competencia, o la habilidad de proveer un cuidado bueno y efectivo; y (4) responsividad (responsiveness) ${ }^{5}$, o la consideración de la situación del otro como él la ve y el reconocimiento del potencial de abuso en el cuidado (Tronto, 1994). Al cuidar de otros, nos orientamos hacia ellos en nuestra atención, nuestras emociones y nuestras acciones.

Ahora bien, cuidar de otros no implica que actuemos siempre según lo que ellos quieren o estiman conveniente, ya que en ocasiones puede ser necesario actuar en contravía de ello, o intentar convencerlos o seducirlos hacia querer algo diferente ${ }^{6}$. Como lo explica Noddings (2005, s. p.):

Cuando cuido, mi energía motivacional comienza a fluir hacia las necesidades y deseos de quien es cuidada/o. Esto no significa que siempre apruebe lo que la/el otra/o desea, ni significa que nunca voy a intentar guiarla/o hacia un mejor conjunto de valores, pero debo tener en cuenta los sentimientos y deseos que están eventualmente allí y responder de la manera más positiva que mis valores y capacidades permitan.

El cuidado implica, pues, responder ante una necesidad de otro, y no solamente ante el deseo del otro. En ese sentido, el cuidado presupone que quien es cuidado puede equivocarse en su deseo. Esto puede implicar una cierta idea de superioridad de quien cuida sobre quien es cuidado, pero solamente en ese momento y en ese lugar, y con relación al asunto alrededor del cual se está actuando. Es decir, reconocer la posibilidad de equivocación no necesariamente implica creer que quien es cuidado siempre está equivocado y que quien cuida siempre puede darse cuenta de la necesidad de quien es cuidado, sin posibilidad de equivocación. Por esto, este reconocimiento de falibilidad debe darse en los dos sentidos: tanto quien

5. A falta de una palabra adecuada en castellano, opté por castellanizar la palabra inglesa que usa Noddings: responsiveness. Se refiere a una disposición y capacidad para responder ante una necesidad. Las traducciones del diccionario, sensibilidad y receptividad, no connotan la acción que trae la palabra inglesa.

6. En este sentido, el cuidado no implica lo que Kohlers (2016) llama deferencia, o actuar como el otro lo haría si pudiera estar en dos lugares al mismo tiempo, la cual para él es central en su concepción de solidaridad. 
cuida como quien es cuidado deben partir del supuesto de que ambos pueden fallar en percibir correctamente la necesidad y la mejor forma de responder a ella.

Propuse arriba que la relación educativa en la pedagogía crítica sea entendida como una relación de cuidado. Esto implica, entonces, que el educador debe estar permanentemente atento a las necesidades de los educandos, y a partir de su juicio contextual, situado, y por lo tanto de un modo fronético, pero también atento a su propia falibilidad, decidir en cada caso y según lo valore más conveniente en un sentido ético, si eventualmente en alguna situación deba primar uno de los dos afanes mencionados antes —el afán de igualdad y el afán de justicia - sobre el otro, o si es posible reconciliarlos y permitir que ambos se desarrollen de manera armónica.

Esto no significa que se establezca una relación de dependencia. Por un lado, en cada situación puede producirse una interacción de modo diferente, precisamente porque no se trata de seguir un principio general. Por esto, la relación puede ser multivalente y cambiante. Y, por otro lado, el cuidado puede también darse en doble vía: en unas situaciones yo seré la persona cuidada y otra la cuidadora, mientras que en otras se invertirán los papeles de cuidado. Por supuesto, esta imagen sugiere una relación diferente de la que posiblemente se manifiesta en el trabajo muchos educadores críticos.

Este ejercicio amoroso y contextualizado, particular más que general, quiero proponer, se encuentra en el centro de la pedagogía. Como tal, constituye un dilema incorporado en la práctica pedagógica misma que nos recuerda lo inapropiado de buscar principios generales que simplemente se apliquen a cada caso o situación concreta en la práctica educativa. Aquí, no hablamos ya del dilema en el que se encuentra el educando — como lo planteaba Buckingham - sino del dilema en que se encuentra constantemente el educador crítico. Un dilema inescapable que demanda su mejor juicio en cada momento y para el cual no puede simplemente recurrir a principios generales.

\section{REFERENCIAS BIBLIOGRÁFICAS}

Burbules, N. (1993). Dialogue in Teaching: Theory and Practice. Nueva York: Teachers College. Burbules, N. (1995). Forms of Ideology-Critique: A Pedagogical Perspective. En P. McLaren y J. Giarelli (Eds.), Critical Theory and Educational Research (pp. 53-69). Nueva York: State University of New York.

Callan, E. (2006). Love, Idolatry, and Patriotism. Social Theory and Practice, 32(4), 525-546.

Ellsworth, E. (1989). Why Doesn't This Feel Empowering? Working Through the Repressive Myths of Critical Pedagogy. Harvard Educational Review, 59(3), 297-324.

Freire, P. (1997). Educación como práctica de la libertad. Bogotá: Siglo XXI.

Freire, P. (2000). Pedagogía del oprimido. Bogotá: Siglo XXI.

Freire, P. (2007). Pedagogía de la esperanza. Bogotá: Siglo XXI.

Giroux, H. (1983). Ideology and Agency in the Process of Schooling. En, Pedagogy and the Politics of Hope: Theory, Culture, and Schooling (pp. 71-94). Boulder: Westview. 
Giroux, H. (1989). Border Pedagogy in the Age of Postmodernism. En, Pedagogy and the Politics of Hope: Theory, Culture, and Schooling (pp. 147-163). Boulder: Westview.

Green, B. (1998). Teaching for Difference: Learning Theory and Post-critical Pedagogy. En D. Buckingham (ed.), Teaching Popular Culture. Beyond Radical Pedagogy (pp. 177-197). Londres: UCL Press.

Hodgson, N., Vlieghe, J. y Zamojski, P. (2017). Manifesto for a Post-critical Pedagogy. London, Punctum Books. Recuperado de: https://punctumbooks.com/titles/manifesto-for-a-postcritical-pedagogy/ (Consultado el 25/03/2020).

Kohlers, A. (2016). A Moral Theory of Solidarity. Oxford: Oxford University Press.

Lyotard, J.F. (1988). The Differend. Manchester: Manchester University Press.

Mejía, A. (2004). The Problem of Knowledge Imposition: Paulo Freire and Critical Systems Thinking. Systems Research and Behavioral Science, 21(1), 63-82.

Mejía, A. (2009). Studying Knowledge Imposition in Pedagogy: A Critical Systemic Framework. Saärbrucken: VDM Verlag.

Noddings, N. (2003). Caring: A Feminine Approach to Ethics and Moral Education. Berkeley: University of California Press.

Noddings, N. (2005). Caring in education. The Encyclopedia of Informal Education. Recuperado de: http://infed.org/mobi/caring-in-education/ (Consultado el 25/03/2020).

Rancière, J. (2003). El maestro ignorante. Cinco lecciones sobre la emancipación intelectual. Barcelona: Laertes.

Rattansi, A. y Reeder, D. (Eds.) (1992). Rethinking Radical Education. Londres: Lawrence and Wishart.

Segato, R. (2016). La guerra contra las mujeres. Madrid: Traficantes de Sueños.

Spivak, G. C. (1988). Can the Subaltern Speak? En C. Nelson y L. Grossberg (Eds.), Marxism and the Interpretation of Culture (pp. 271-313). Basingstoke: MacMillan.

Tronto, J. (1994). Moral Boundaries: A Political Argument for an Ethic of Care. Nueva York: Routledge.

Tronto, J. (2006). Women and Caring: What Can Feminists Learn about Morality from Caring? En V. Held (Ed.), Justice and Care: Essential Readings in Feminist Ethics (pp. 101-115). Boulder: Westview Press. 